\title{
In-Situ and Ex-situ TEM Imaging and Spectroscopy Study of Li-Ion Battery
}

C. M. Wang ${ }^{*}$, W. Xu ${ }^{* *}$, Z. M. Nie ${ }^{* *}$, D. Choi ${ }^{* *}$,D. H. Wang ${ }^{* * *}$,

Z. G. Yang ${ }^{* *}$, J. G. Zhang ${ }^{* *}$, S. Thevuthasan, , J. Liu ${ }^{* * *}$, and D. R. Baer

${ }^{*}$ Environmental Molecular Sciences Laboratory, Pacific Northwest National Laboratory, Richland, WA 99352, USA

** Energy and Environmental Directorate

Pacific Northwest National Laboratory, Richland, WA 99352, USA

${ }^{* * *}$ Fundamental and Computational Science Directorate

Pacific Northwest National Laboratory, Richland, WA 99352, USA

Although Li-ion battery technology has been identified as having great potential for energy storage, a lack of basic understanding of the structural and chemical evolution of the materials during the operation of the battery [1] is slowing the rate of battery development. Repeated charging and discharging of a Li-ion battery induces microstructural changes at the interface between the electrolyte and the electrode and within the electrode (active materials) due to Li migration. Although it has been established that this structural evolution of active materials is responsible for the failure of the battery, the mechanisms of the microstructural changes as a function of charging/discharging are not well understood.

Advanced diagnostic tools such as electron microscopy along with other surface and bulk sensitive tools, often in ex-situ mode, have been used to probe materials changes. However, it is recognized that characterizing this interface using an ex-situ tools will present major challenges for materials and interfaces that are not stable upon exposure to air and many not be stable without the potentials and environmental conditions that occur during battery operation. Capabilities that enable the in situ observation of the structural and chemical changes during the dynamic operation of battery are needed for addressing this scientific challenge. The main objective the work reported is to develop the fundamental scientific understanding of the chemical and structural evolution at the interface between the electrolyte and the electrode as well as within the electrodes under the dynamic (Li charging and discharging) operating conditions.

Electron energy-loss spectroscopy (EELS) in a TEM not only allows the direct detection of Li following the lithiation, but also provides information related to local electronic structure of the materials with a spatial resolution from several nanometers to single atomic column. EELS operated in a spectroscopic imaging mode will allow the mapping of the distribution of a specified chemical species with a spatial resolution of nanometer to atomic scale. In spite of the great interests in developing and characterizing novel materials for energy storage in battery applications, up to date EELS techniques have not been widely used to study the lithium insertion in electrode materials used in Li-ion batteries. For Li insertion into the lattice of $\mathrm{TiO}_{2}$, the detection of $\mathrm{Li}$ has been often carried out indirectly, mostly based on the phase and structural analysis. Direct detection of $\mathrm{Li}$ in the lattice of $\mathrm{TiO}_{2}$ and investigation of the associated electronic structure changes due to the $\mathrm{Li}$ incorporation in $\mathrm{TiO}_{2}$ has not been done.

In this paper, we use TEM imaging, electron diffraction, and EELS to probe the microstructure and Li insertion, and to investigate the electronic structure of rutile $\mathrm{TiO}_{2}$ 
before and after the Li insertion by a mechanical activation method. The EELS results provide direct information about electronic structure and the charge transfer in lithiated $\mathrm{TiO}_{2}$. This data provides the baseline information useful for direct observation of $\mathrm{Li}$ incorporation during battery cycling [2].

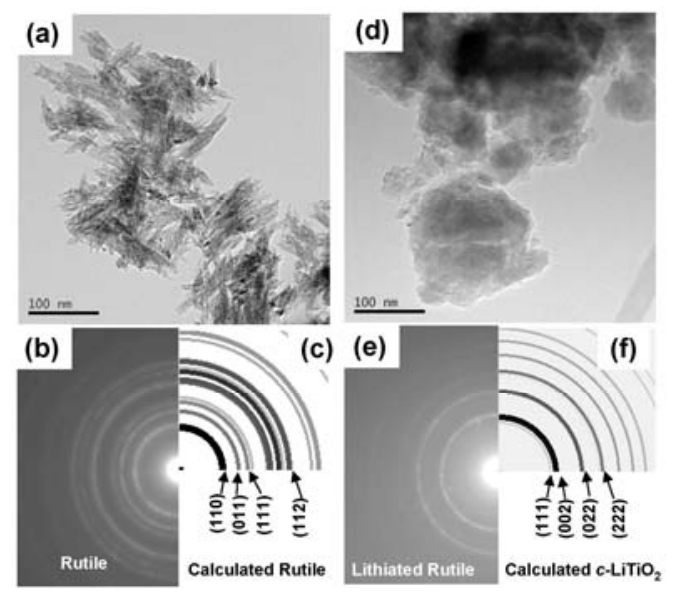

Fig. 1. TEM images and electron diffraction analysis of the commercial $\mathrm{TiO}_{2}$ particles before and after lithiation. (a) Bright-field TEM image of the as-received $\mathrm{TiO}_{2}$. (b) Selected area electron diffraction pattern of the $\mathrm{TiO}_{2}$. (c) Calculated electron diffraction ring pattern of $\mathrm{TiO}_{2}$ rutile. (d) Bright-field TEM image of the mechanically lithiated $\mathrm{TiO}_{2}$ powder particle shown in (a). (e) Selected area electron diffraction pattern of powder particles shown in (d). (f) Calculated electron diffraction ring pattern of cubic $\mathrm{LiTiO}_{2}$.

The microstructural changes of $\mathrm{TiO}_{2}$ before and after the lithiation as well as the corresponding electron diffraction pattern were shown in Figure 1. Mechanical milling of these powder particles with Li metal particles induced significant morphological changes. After the milling, the needle-shaped primary particles are no longer visible. Instead, the lithiated powder is aggregates of round-shaped particles. EELS measurements reveal the lithiated $\mathrm{TiO}_{2}$ a characteristic Li K-edge at the energy-loss position of $61 \mathrm{eV}$. EELS fine structural features of both $\mathrm{Ti} \mathrm{L}_{2,3}$-edge and $\mathrm{O} \mathrm{K}$-edge suggest charge transfer from Li to Ti during the lithiation via mechanical activation. This is consistently supported by the electron diffraction analysis on the formation of rock-salt structured $\mathrm{LiTiO}_{2}$.

Acknowledgements: This work was supported U.S. Department of Energy (DOE) Office of Science, Offices of Basic Energy Sciences and Biological and Environmental Research. The work was conducted in the William R. Wiley Environmental Molecular Sciences Laboratory (EMSL) a DOE User Facility operated by Battelle for the DOE Office of Biological and Environmental Research. Pacific Northwest National Laboratory is operated for the DOE under Contract DE-AC06-76RLO 1830.

\section{References}

1. C. H. Jiang, I. Honma, T. Kudo, and H. S. Zhou, Electrochem. Solid-State Lett., 10 (2007) A127.

2 C. M. Wang, Z. G. Yang, S. Thevuthasan, J. Liu, D. R. Baer, D. Choi, D. H. Wang, J. G. Zhang, L. Saraf, and Z. M. Nie, Appl. Phy. Lett., under review, 2008. 